(c) 1999 International Press

Adv. Theor. Math. Phys. 3 (1999) 227-248

\title{
M theory, Joyce Orbifolds and Super Yang-Mills.
}

\author{
B.S. Acharya \\ Department of Physics, \\ Queen Mary and Westfield College, \\ Mile End Road, \\ Londion. E1 4NS. \\ r.acharya@qmw . ac . uk
}

\begin{abstract}
We geometrically engineer $d=4 \mathrm{~N}=1$ supersymmetric Yang-Mills theories by considering $M$ theory on various Joyce orbifolds. We argue that the superpotential of these models is generated by fractional membrane instantons. The relation of this superpotential to membrane anomalies is also discussed.
\end{abstract}

e-print archive: http://xxx.lanl.gov/hep-th/9812205

Work supported by PPARC 


\section{Introduction}

In recent years string theory and $M$ theory have provided us with a deeper insight into the dynamics of (mainly supersymmetric) gauge theories in various dimensions. Difficult questions in field theory can turn out to have relatively simple answers when embedded into a string or $M$ theoretic context.

This powerful approach to the study of gauge theories comes, roughly, in two related forms: one can study physics localised on branes by decoupling "bulk degrees of freedom" or one can consider the physics localised near certain kinds of singularities in spacetime, a construction which goes by the name of geometric engineering. Constructions of the former type were initiated in [1] building on results of many other authors and are reviewed in [2], whereas geometric engineering was christened in [3] a paper which was also motivated by the results of several authors concerning massless states appearing at singularities. Many references to these works can be found in [3] and [4].

$M$ theory compactified on a 7-manifold with $G_{2}$ holonomy gives rise to a four dimensional theory with $\mathcal{N}=1$ supersymmetry. For a given 7-manifold $\mathbf{X}$ with $G_{2}$ holonomy, what is the physics of $M$ theory compactified on $\mathbf{X}$ ? At low energies we can use the eleven dimensional supergravity approximation to $M$ theory and one finds that the effective four dimensional supergravity theory describing the massless modes, as first noted in [5], is $\mathcal{N}=1$ supergravity coupled to $b_{2} U(1)$ vector multiplets and $b_{3}$ neutral chiral multiplets, where $b_{i}$ are Betti numbers of $\mathbf{X}$. Such a supergravity theory is relatively uninteresting physically. However, this particular massless spectrum, is that of $M$ theory compactified on a smooth 7-manifold of $G_{2}$ holonomy. On the other hand, an important lesson learned in string theory and $M$ theory over the past few years has been that interesting physics can arise when singularities in a "compactification manifold" appear. The main purpose of this paper is to initiate a study of the physics associated with singularities in spaces with $G_{2}$ holonomy. In particular, we will construct and study 'local models' of natural (and perhaps the simplest) types of singularities in this context; we will use these models to geometrically engineer four dimensional $\mathcal{N}=1$ supersymmetric Yang-Mills theories. Geometric engineering of $\mathcal{N}=1$ theories has been studied in detail previously in the context of Calabi-Yau backgrounds [6].

To motivate the types of singularities we will study consider the following. Let $\mathbf{S}$ be a $\mathbf{K 3}$ surface and $\left\{\omega_{i}\right\}$ a triplet of Kahler forms defining a hyperkahler structure on $\mathbf{S}$. Let $\mathbf{T}^{\mathbf{3}}$ be a flat 3 -torus and $d x_{i}$ a basis for its 
cotangent bundle. Then the product manifold $\mathbf{S} \times \mathbf{T}^{\mathbf{3}}$ admits a parallel $G_{2}$ structure defined as follows.

$$
\begin{aligned}
\varphi & =\delta^{i j} \omega_{i} \wedge d x_{j}+\frac{1}{6} \epsilon^{i j k} d x_{i} \wedge d x_{j} \wedge d x_{k} \\
\star \varphi & =\frac{1}{6} \delta^{i j} \omega_{i} \wedge \omega_{j}+\frac{1}{2} \epsilon^{i j k} \omega_{i} \wedge d x_{j} \wedge d x_{k}
\end{aligned}
$$

In the above $\delta^{i j}$ is the identity matrix, $\epsilon^{123}=1$ and is totally antisymmetric and we have used the Einstein summation convention. $S O(3)$ acts naturally on both triplets $\omega_{i}$ and $d x_{i}$. The above $G_{2}$ structure is hence manifestly $S O(3)$-invariant.

Let $\mathbf{K}$ denote a finite subgroup of $S O(3)$ under which the triplets $\omega_{i}$ and $d x_{i}$ transform in the same representation. Then the orbifold $\mathbf{O}_{\mathbf{K}} \equiv$ $\left(\mathbf{S} \times \mathbf{T}^{\mathbf{3}}\right) / \mathbf{K}$ inherits the above parallel $G_{2}$ structure. Furthermore, if $\mathbf{K}$ is not a subgroup of $S O(2) \subset S O(3)$, then $\mathbf{O}_{\mathbf{K}}$ will not inherit a parallel $S U(3)$ structure. We will always choose $\mathbf{K} \not \subset S O(2)$ in what follows. This technical restriction ensures $\mathcal{N}=1$ supersymmetry and not $\mathcal{N}=2$.

If we now consider $M$ theory on $\mathbf{O}_{\mathbf{K}} \times \mathbf{R}^{\mathbf{4}}$, then we obtain an effective four dimensional theory with $\mathcal{N}=1$ supersymmetry (assuming as we will do that such an orbifold compactification is well defined in $M$ theory). There are now essentially four cases to consider. (i) $\mathbf{K}$ acts freely on $\mathbf{S} \times \mathbf{T}^{\mathbf{3}}$ and $\mathbf{S}$ is a non-singular $\mathbf{K 3}$ surface; (ii) $\mathbf{K}$ acts freely on $\mathbf{S} \times \mathbf{T}^{\mathbf{3}}$ and $\mathbf{S}$ is a singular $\mathbf{K 3}$ surface; (iii) $\mathbf{K}$ does not act freely and $\mathbf{S}$ is non-singular; (iv) $\mathbf{K}$ does not act freely and $\mathbf{S}$ is singular. By singular, we mean a K3 surface with A-D-E orbifold singularities, so that the notion of hyperkahler structure still makes sense. In case $(i) \mathbf{O}_{\mathbf{K}}$ is a smooth manifold and therefore the low energy physics is that we described above. $\mathbf{O}_{\mathbf{K}}$ clearly has singularities in the remaining three cases. In this paper we will describe the physics localised near singularities of type (ii). We hope to address types (iii) and (iv) in future investigations.

For cases of type (ii) the space $\mathbf{O}_{\mathbf{K}}$ can naturally be thought of as being fibered by the $\mathbf{K 3}$ surfaces $\mathbf{S}$. K3 fibrations of Joyce orbifolds [7] with $G_{2}$ holonomy were discussed in the context of $M$ theory/heterotic duality in [8] and have been further investigated in [9].

We are interested ultimately in $M$ theory physics near the singularities of $\mathbf{O}_{\mathbf{K}}$. Since in the case of type (ii) these correspond locally to A-D-E surface singularities, in order to construct a local model we will 'replace' $\mathbf{S}$ with an orbifold $\mathbf{C}^{2} / \mathbf{G}$, where $\mathbf{G}$ is a finite subgroup of $S U(2)$. In other words we will be considering orbifolds of the form $\mathbf{J}_{\mathbf{K}}^{\mathrm{G}} \equiv\left(\mathbf{C}^{\mathbf{2}} / \mathbf{G} \times \mathbf{T}^{\mathbf{3}}\right) / \mathbf{K}$. By abuse 
of notation, if we let $\omega_{i}$ denote the hyperkahler structure on $\mathbf{C}^{2} / \mathbf{G}$, then equations (1) and (2) define a parallel $G_{2}$-structure on $\mathbf{J}$.

We can further divide the orbifolds $\mathbf{J}$ into two types. Since $\mathbf{K}$ acts freely on $\mathbf{C}^{\mathbf{2}} / \mathbf{G} \times \mathbf{T}^{\mathbf{3}}$, these differ according to whether or not $\mathbf{K}$ acts freely on $\mathbf{T}^{\mathbf{3}}$. We will only consider cases in which $\mathbf{K}$ does act freely on $\mathbf{T}^{\mathbf{3}}$, in which case $\mathbf{J}$ is naturally endowed with the structure of a fibration whose fibers are the $\mathbf{C}^{\mathbf{2}} / \mathbf{G}$ orbifolds and whose base is the smooth 3 -manifold $\mathbf{T}^{\mathbf{3}} / \mathbf{K}$. The singular set of the orbifold is $\mathbf{M}_{\mathbf{K}} \equiv\left(\{\mathbf{0}\} \times \mathbf{T}^{\mathbf{3}}\right) / \mathbf{K}$ (where $\{\mathbf{0}\}$ is the origin in $\mathbf{C}^{\mathbf{2}} / \mathbf{G}$ ) and we can see that the singular set of $\mathbf{J}$ consists of a family of A-D-E singularities fibered over $\mathbf{T}^{\mathbf{3}} / \mathbf{K}$.

In the next section we will study families of A-D-E singularities and compute the four dimensional massless spectrum localised near these singularities. The results of this section are in a similar spirit to results which have appeared in $[10,11,3]$ concerning families of A-D-E singularities in Calabi-Yau spaces. We then describe some explicit examples of the orbifolds $\mathbf{J}_{\mathbf{K}}^{\mathbf{G}}$ for which $\mathbf{G}$ is any finite A-D-E subgroup of $S U(2)$ and $\mathbf{K}$ is isomorphic to $\mathbf{Z}_{\mathbf{2}} \times \mathbf{Z}_{\mathbf{2}}$ or $\mathbf{Z}_{\mathbf{2}} \ltimes \mathbf{Z}_{\mathbf{4}}$. It turns out that the geometrically engineered models obtained from $M$ theory on $\mathbf{J}_{\mathbf{K}}^{\mathbf{G}}$ are described at low energies by pure $\mathcal{N}=$ 1 super Yang-Mills theory with A-D-E gauge group corresponding to $\mathbf{G}$.

We then take a slight detour and propose a Type IIA dual description of some of the $M$ theory models involving D6-branes. This section also clarifies certain aspects of the computation of the spectrum in section two. Following a brief review of $\mathcal{N}=1$ super Yang-Mills theory (which is included mainly for completeness) we discuss the $\frac{1}{2}$ supersymmetric BPS states present in the geometrically engineered models. We show firstly that the models engineered using $\mathbf{J}_{\mathbf{K}}^{\mathbf{G}}$ contain fractional $M 2$-brane instantons (of a type discussed in [12]), which correspond in field theory to states with fractional instanton numbers; secondly we argue that fractional instantons of a particular topological charge can generate a superpotential. This superpotential matches precisely that expected from field theory considerations. In [13] it was argued that fractional instantons generate the superpotential in an alternative construction [14] (now known as $M$ QCD) of the same field theory (with gauge group $S U(n)$ ) using Type IIA/ $M$ branes. We refer the reader to [2] for further references to $\mathcal{N}=1$ models constructed using branes. Furthermore, it has been argued in [16] in field theory and in [17] in the geometrically engineered models of [6] that such a superpotential can be generated by torons in a certain limit. Several other aspects of fractional instantons in field theory have also been discussed in [18].

In the final section of this paper we describe a relation between the 
instanton generated superpotential and the parity anomaly on the $M 2$-brane world-volume. The result here is that, modulo an assumption, $M 2$-branes indeed know about the anomaly seen in the field theory. This is a satisfying confirmation of the main results which complement nicely the results of [23] in which superpotentials were generated using $M 5$-brane instantons in Calabi-Yau spaces.

Note Added. After completing the first version of this paper we were informed by G. Moore of the forthcoming paper [25] in which there is overlap with the present work.

\section{Supersymmetric Families of A-D-E singularities}

The orbifolds $\mathbf{J}_{\mathbf{K}}^{\mathrm{G}}$ can be thought of as families of $\mathbf{C}^{2} / \mathbf{G}$ orbifolds parameterised by $\mathbf{M}_{\mathbf{K}}$. $\mathbf{M}_{\mathbf{K}}$ is naturally a supersymmetric 3-cycle. This is straightforward to establish since $\{\mathbf{0}\} \times \mathbf{T}^{\mathbf{3}}$ is a supersymmetric cycle in $\mathbf{C}^{\mathbf{2}} / \mathbf{G} \times \mathbf{T}^{\mathbf{3}}$ and $\mathbf{K}$ preserves its volume form. Therefore we are considering the orbifold singularities of $\mathbf{J}_{\mathbf{K}}^{\mathbf{G}}$ as local models for supersymmetric (ie associative) families of A-D-E surface singularities which might develop in spaces of $G_{2}$ holonomy as we move through the moduli space of metrics.

What is the $M$ theory physics localised near the singularities of $\mathbf{J}_{\mathbf{K}}^{\mathbf{G}}$ ? In order to answer this question, we should first compute what massless modes are supported near the singularities. We will actually give a more general answer which computes the massless spectrum localised near any 3-dimensional supersymmetric family of A-D-E singularities. Therefore we consider a manifold $\mathbf{X}$ with $G_{2}$ holonomy. As we vary the $G_{2}$ holonomy metrics on $\mathbf{X}$ we assume that we encounter a 3-dimensional family of A-D-E singularities parametrised by a 3 -manifold $\mathbf{M}$ such that $\mathbf{M}$ is a supersymmetric submanifold of the singular limit of $\mathbf{X}$.

Begin first by considering $M$ theory near an A-D-E singularity of the form $\{\mathbf{x}\} \times \mathbf{R}^{\mathbf{7}}$, where $\{\mathbf{x}\}$ is the singular point in a 4-space with hyperkahler structure. The physics localised near such a singularity of spacetime is described by 7-dimensional super Yang-Mills theory on $\mathbf{R}^{\mathbf{7}}$ with A-D-E gauge group $H$ (determined by which A-D-E subgroup of $S U(2) \mathbf{G}$ is). The singularities of spacetime we wish to consider are locally of the form $\{\mathbf{x}\} \times \mathbf{M} \times \mathbf{R}^{\mathbf{4}}$ which occur inside a spacetime $\mathbf{X} \times \mathbf{R}^{4}$ with $\mathbf{X}$ a space of $G_{2}$ holonomy. From the point of view of the 7-dimensional Yang-Mills theory we have essentially replaced $\mathbf{R}^{\mathbf{7}}$ with $\mathbf{M} \times \mathbf{R}^{\mathbf{4}}$. We should thus consider 7-dimensional super Yang-Mills theory on $\mathbf{M} \times \mathbf{R}^{4}$ and compute the massless modes in $\mathbf{R}^{4}$ a la 


\section{Kaluza-Klein.}

Since generically $\mathbf{M}$ will not admit any parallel spinors one might first think that all supersymmetry is broken by this compactification. However, $\mathbf{M}$ is a supersymmetric 3-cycle in a space with $G_{2}$ holonomy and this means that the Yang-Mills theory is twisted and is indeed supersymmetric. This is very much in the spirit of discussions in $[10,19]$. We will illustrate this twisting shortly.

In $\mathbf{R}^{\mathbf{7}}$ the symmetries of super Yang-Mills theory include $\operatorname{Spin}(3) \times \operatorname{Spin}(6,1)$. $\operatorname{Spin}(3)$ is the R-symmetry group and $\operatorname{Spin}(6,1)$ the cover of the Lorentz group. Spin(3) also acts as rotations of the hyperkahler structure associated with the A-D-E singularity. The fields of the theory are all massless and consist of three scalars $\phi_{i}$ transforming in the $(\mathbf{3}, \mathbf{1})$, gauge bosons transforming in the $(\mathbf{1}, 7)$ and 16 fermions in the $(\mathbf{2}, \mathbf{8})$. When we 'compactify' to $\mathbf{M} \times \mathbf{R}^{\mathbf{4}}$ the $\operatorname{Spin}(6,1)$ symmetry is broken to

$\operatorname{Spin}(3)^{\prime} \times S L(2, \mathbf{C})$. Here $S p i n(3)^{\prime}$ is the cover of the structure group of $\mathbf{M}$ and $S L(2, \mathbf{C})$ is the cover of the Lorentz group in 4-dimensions. However, as is perhaps evident in equations (1) and (2), the fact that $\mathbf{M}$ is a supersymmetric submanifold inside a space with $G_{2}$ holonomy means that the theory is 'twisted': in fact the two $\operatorname{Spin}(3)$ groups are identified under this twisting. In this 'twisted' theory the symmetry group is just $S O(3) \times S L(2, \mathbf{C})$ with $S O(3)$ the structure group of $\mathbf{M}$. We give an alternative and clearer description of this twisting in terms of wrapped D6-branes in Type IIA theory later in this paper.

The fields and their transformation properties under this twisted symmetry group are, in the bosonic sector two 1-forms on $\mathbf{M}$ which are scalars in $\mathbf{R}^{\mathbf{4}}$. These transform as $\mathbf{2}(\mathbf{3}, \mathbf{1})$. There are also scalars on $\mathbf{M}$ which are gauge fields on $\mathbf{R}^{\mathbf{4}}$; these transform as $(\mathbf{1}, \mathbf{2} \otimes \overline{\mathbf{2}})$. The fermions of the theory are organised in the following fashion. There are two spacetime fermions one of which is a scalar on $\mathbf{M}$ the second of which is a 1-form on $\mathbf{M}$. The fermions thus transform as $(\mathbf{1}+\mathbf{3}, \mathbf{2}+\overline{\mathbf{2}})$. These fields will be massless if they are zero modes of the relevant derivative operator on $\mathbf{M}$. In this case the relevant operator is just the Laplacian on $\mathbf{M}$.

We thus find that the massless spectrum supported near this family of singularities consists of 1 gauge field, $b_{1}$ scalars and $1+b_{1}$ fermions. $\left(b_{1}\right.$ is the first Betti number of $\mathbf{M}$ ). We should also recall that in the uncompactified theory all the fields transform in the adjoint representation of the A-D-E gauge group. This however, does not imply that the gauge group of the four dimensional theory is the same as that in seven dimensions; as noted in the context of $F$-theory, when obtaining lower dimensional gauge fields 
from higher dimensions by 'fibering' A-D-E singularities over some space $\mathbf{B}$, certain monodromies in the fibration can act as outer automorphisms of the A-D-E group and hence break the gauge group to the subgroup commuting with this automorphism. In this paper we will mainly consider situations in which this does not occur. Whether or not such a monodromy occurs depends upon the choice of the group $\mathbf{K}$ in the spaces $\mathbf{J}_{\mathbf{K}}^{\mathbf{G}}$. If we assume that such outer automorphisms do not occur then all the above massless fields transform in the adjoint representation of the A-D-E gauge group. Otherwise they transform under the broken gauge group. The massless field content is then precisely that of pure $\mathcal{N}=\left(1+b_{1}\right)$ super Yang-Mills theory in four dimensions.

For the orbifolds $\mathbf{J}_{\mathbf{K}}^{\mathbf{G}}, \mathbf{M}$ is $\mathbf{T}^{\mathbf{3}} / \mathbf{K}$ and has $b_{1}=0$. Hence the massless spectrum consists of pure $\mathcal{N}=1$ super Yang-Mills theory with A-D-E gauge group determined by $\mathbf{G}$.

As a check of this result consider the special cases when $\mathbf{K}$ is the trivial

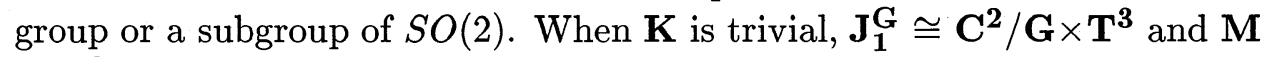
$\cong \mathbf{T}^{\mathbf{3}}$. Hence our computation correctly predicts that the massless modes supported near the singularity are those of pure $\mathcal{N}=4$ super Yang-Mills theory. In the case when $\mathbf{K}$ is a subgroup of $S O(2)$ and $\mathbf{J}_{\mathbf{K}}^{\mathbf{G}}$ admits a parallel $S U$ (3) structure, $\mathbf{M} \cong \mathbf{C P}^{\mathbf{1}} \times \mathbf{S}^{\mathbf{1}}$ and our computation correctly predicts a spectrum $[10,11]$ which matches that of pure $\mathcal{N}=2$ super Yang-Mills theory.

For concreteness we introduce some particular examples of spaces with the properties we require, namely a well defined $G_{2}$ holonomy structure and an associative family of $\mathbf{C}^{\mathbf{2}} / \mathbf{G}$ hyperkahler orbifold singularities. In fact, for these examples, $\mathbf{K} \cong \mathbf{Z}_{\mathbf{2}} \times \mathbf{Z}_{\mathbf{2}}$ or $\mathbf{Z}_{\mathbf{2}} \times \mathbf{Z}_{\mathbf{4}}$ and we can define one such space for each A-D-E subgroup of $S U(2)$.

\section{$2.1 \quad$ Examples}

Our first example orbifolds $\mathbf{J}_{\mathbf{Z}_{2} \times \mathbf{Z}_{2}}^{\mathbf{G}}$, are defined as follows. We begin with the case $\mathbf{G} \cong \mathbf{Z}_{\mathbf{n}}$ and $\mathbf{R}^{\mathbf{7}} \equiv \mathbf{C}^{\mathbf{2}} \times \mathbf{R}^{\mathbf{3}}$. Let $\left(z_{1}, z_{2}\right)$, and $x_{i}(i=1,2,3)$ denote coordinates for the $\mathbf{C}^{\mathbf{2}}$ and $\mathbf{R}^{\mathbf{3}}$ factors respectively. Form $\mathbf{C}^{\mathbf{2}} \times \mathbf{T}^{\mathbf{3}}$ by identifying:

$$
\left(x_{i}\right) \equiv\left(x_{i}+1\right)
$$

Define three finite groups of isometries of the above $\mathbf{C}^{\mathbf{2}} \times \mathbf{T}^{\mathbf{3}}$, which are respectively isomorphic to $\mathbf{Z}_{\mathbf{n}}, \mathbf{Z}_{\mathbf{2}}$ and $\mathbf{Z}_{\mathbf{2}}$ and which together generate $\boldsymbol{\Gamma} \cong$ $\left(\mathbf{Z}_{2} \times \mathbf{Z}_{2}\right) \ltimes \mathbf{Z}_{\mathbf{n}}$ :

$$
\mathbf{Z}_{\mathbf{n}}(\alpha):\left(z_{1}, z_{2}, x_{1}, x_{2}, x_{3}\right)=\left(e^{2 \pi i / n} z_{1}, e^{-2 \pi i / n} z_{2}, x_{1}, x_{2}, x_{3}\right)
$$




$$
\begin{aligned}
& \mathbf{Z}_{\mathbf{2}}(\beta):\left(z_{1}, z_{2}, x_{1}, x_{2}, x_{3}\right)=\left(-z_{1}, z_{2},-x_{1}+\frac{1}{2},-x_{2}, x_{3}+\frac{1}{2}\right) \\
& \mathbf{Z}_{\mathbf{2}}(\gamma):\left(z_{1}, z_{2}, x_{1}, x_{2}, x_{3}\right)=\left(-z_{1}^{*},-z_{2}^{*},-x_{1}, x_{2}+\frac{1}{2},-x_{3}\right)
\end{aligned}
$$

We have denoted by $(\alpha, \beta, \gamma)$ the generators of the three cyclic groups and * denotes complex conjugation.

The orbifold $\mathbf{J}_{\mathbf{Z}_{\mathbf{2}} \times \mathbf{Z}_{\mathbf{2}}}^{\mathbf{Z}_{\mathbf{2}}}$ is defined as the quotient $\left(\mathbf{C}^{\mathbf{2}} \times \mathbf{T}^{\mathbf{3}}\right) / \boldsymbol{\Gamma}$.

Let $\mathbf{F}$ be the subgroup of $\boldsymbol{\Gamma}$ which acts with fixed points and denote by $\boldsymbol{\Gamma}^{\prime}$ the quotient group $\boldsymbol{\Gamma} / \mathbf{F}$. Then it is easy to see that $\mathbf{F} \cong \mathbf{Z}_{\mathbf{n}}$ (generated by $\alpha$ ) and $\boldsymbol{\Gamma}^{\prime} \cong \mathbf{Z}_{\mathbf{2}} \times \mathbf{Z}_{\mathbf{2}}$ (generated by $\beta \mathbf{Z}_{\mathbf{n}}$ and $\gamma \mathbf{Z}_{\mathbf{n}}$ ). $\boldsymbol{\Gamma}^{\prime}$ acts freely on $\mathbf{C}^{\mathbf{2}} \times \mathbf{T}^{\mathbf{3}}$; therefore the singular set of the orbifold $\mathbf{J}$ consists of $\{0\} \times \mathbf{T}^{\mathbf{3}} / \boldsymbol{\Gamma}^{\prime}$, where $\{0\}$ is the origin in $\mathbf{C}^{\mathbf{2}}$. The singular set is thus a $\mathbf{T}^{\mathbf{3}} / \boldsymbol{\Gamma}^{\prime}$ family of $A_{n-1}$ singularities.

There is one subtlety pertaining to these examples: there is a non-trivial monodromy of the singularity for $n \geq 2$. This is due to the group relation

$$
\gamma \alpha^{k} \gamma^{-1}=\alpha^{-k}
$$

More generally, if we replace $\mathbf{Z}_{\mathbf{n}}$ in the above with any of the finite A-DE subgroups of $S U(2)$, but keep the group $\mathbf{Z}_{\mathbf{2}} \times \mathbf{Z}_{\mathbf{2}}$ fixed, we obtain a space $\mathbf{J}_{\mathbf{Z}_{2} \times \mathbf{Z}_{2}}^{\mathbf{G}}$ whose singularities are a family of A-D-E singularities fibered over $\mathbf{M}_{\mathbf{Z}_{\mathbf{2}} \times \mathbf{Z}_{\mathbf{2}}}$. For the cases corresponding to $\mathrm{D}$ and $\mathrm{E}$ gauge groups one can in fact check that there is no monodromy corresponding to outer automorphisms of the Lie algebra of the gauge group.

Our second example orbifolds are obtained by taking $\mathbf{K} \cong \mathbf{Z}_{\mathbf{2}} \ltimes \mathbf{Z}_{\mathbf{4}}$. This group is defined to act in the following way on $\mathbf{C}^{\mathbf{2}} \times \mathbf{T}^{\mathbf{3}}$ :

$$
\begin{aligned}
& \mathbf{Z}_{\mathbf{2}}\left(\beta^{\prime}\right):\left(z_{1}, z_{2}, x_{1}, x_{2}, x_{3}\right)=\left(-z_{1}, z_{2},-x_{1},-x_{2}+\frac{3}{4}, x_{3}+\frac{1}{2}\right) \\
& \mathbf{Z}_{4}(\eta):\left(z_{1}, z_{2}, x_{1}, x_{2}, x_{3}\right)=\left(-i z_{2}^{*}, i z_{1}^{*}, x_{1}+\frac{1}{4},-x_{2}+\frac{1}{4},-x_{3}\right)
\end{aligned}
$$

The orbifolds $\mathbf{J}_{\mathbf{Z}_{\mathbf{2}} \ltimes \mathbf{Z}_{\mathbf{4}}}^{\mathbf{G}}$ are defined as above for $\mathbf{G}$ any ADE subgroup of $S U(2)$. In this case one may check that $\mathbf{\Gamma} \cong \mathbf{G} \times\left(\mathbf{Z}_{\mathbf{2}} \ltimes \mathbf{Z}_{\mathbf{4}}\right)$. The singular set of $\mathbf{J}_{\mathbf{Z}_{\mathbf{2}} \ltimes \mathbf{Z}_{\mathbf{4}}}^{\mathbf{G}}$ consists of a family of ADE singularities fibered over $\mathbf{T}^{\mathbf{3}} /\left(\mathbf{Z}_{\mathbf{2}} \ltimes \mathbf{Z}_{4}\right)$. In these examples, because $\mathbf{Z}_{\mathbf{2}} \ltimes \mathbf{Z}_{\mathbf{4}}$ commutes with $\mathbf{G}$, there is no monodromy of the singularity. The cases in which there is a non-trivial monodromy will be discussed in detail in a forthcoming companion paper, since we do not wish to cloud the main points of this article with these issues. 


\section{Relation to D6-branes}

In this section we will relate $M$ theory on $\mathbf{J}_{\mathbf{K}}^{\mathbf{Z}_{\mathbf{n}}}$ to a certain configuration of partially wrapped D6-branes in Type IIA theory. The purpose of this section is really to confirm the computation of the massless spectrum of $M$ theory fields supported near the singularities of $\mathbf{J}$ and give a clearer origin for the twisting of the 7-dimensional gauge theory that we discussed previously. As such this paper can be read independently of this section.

$M$ theory on $\mathbf{C}^{\mathbf{2}} / \mathbf{Z}_{\mathbf{n}} \times \mathbf{R}^{\mathbf{7}}$ gives rise to a supersymmetric $S U(n)$ YangMills theory which is supported near the $A_{n-1}$ singularity; hence this gauge theory lives naturally on $\mathbf{R}^{\mathbf{7}}$. In fact, the same 7-dimensional gauge theory arises as the low energy world-volume theory on $n$ parallel D6-branes in Type IIA string theory on $\mathbf{R}^{\mathbf{1 0}}$. The moduli space of this 7-dimensional super Yang-Mills theory is $\mathbf{S y m}^{\mathbf{n}-\mathbf{1}}\left(\mathbf{R}^{\mathbf{3}}\right)$. From the point of view of the D6-branes, this moduli space is easily understood; since they are parallel they all live at a point in $\mathbf{R}^{\mathbf{3}}$ and hence we have a "splitting" of $\mathbf{R}^{\mathbf{1 0}} \cong$ $\mathbf{R}^{\mathbf{3}} \times \mathbf{R}^{\mathbf{7}}$ corresponding to transverse versus world-volume dimensions. Then $\mathbf{S y m}^{\mathbf{n}-1}\left(\mathbf{R}^{\mathbf{3}}\right)$ is just the moduli space of $n$ particles on $\mathbf{R}^{\mathbf{3}}$ subjected to one constraint which eliminates their center of mass motion. From the point of view of $M$ theory on $\mathbf{C}^{2} / \mathbf{Z}_{\mathbf{n}}$, it is more difficult to understand the gauge theory moduli space since it is related to the moduli space of hyperkahler structures on certain ALE-spaces and their orbifold degenerations. What will be important is the fact that the $S O(3)$ R-symmetry group of the gauge theory acts on the coordinates of the various $\mathbf{R}^{\mathbf{3}}$ factors in the moduli space according to the 3 -dimensional representation. This $S O(3)$ also acts on the hyperkahler orbifold $\mathbf{C}^{\mathbf{2}} / \mathbf{Z}_{\mathbf{n}}$ via rotations of the three Kahler forms on this space.

Since $M$ theory on $\mathbf{C}^{2} / \mathbf{Z}_{\mathbf{n}}$ gives rise to a gauge theory equivalent to that which comes from $n$ D6-branes at a point in $\mathbf{R}^{\mathbf{3}}, M$ theory on $\mathbf{C}^{\mathbf{2}} / \mathbf{Z}_{\mathbf{n}} \times \mathbf{T}^{\mathbf{3}}$ corresponds from the point of view of the D6-branes to world-volumes which are no longer $\mathbf{R}^{\mathbf{7}}$ but rather $\mathbf{T}^{\mathbf{3}} \times \mathbf{R}^{\mathbf{4}}$. This "compactification" of the 7 dimensional D6-brane world-volume does not affect the $\mathbf{R}^{\mathbf{3}}$ to which they are transverse. If we take the 7-dimensional gauge coupling constant to zero, whilst at the same time shrinking the volume of the 3-torus, then we can obtain an effective 4-dimensional super Yang-Mills theory which lives on $\mathbf{R}^{\mathbf{4}}$. This theory has 4-dimensional $\mathcal{N}=4$ supersymmetry. When we further orbifold $M$ theory on $\mathbf{C}^{\mathbf{2}} / \mathbf{Z}_{\mathbf{n}} \times \mathbf{T}^{\mathbf{3}}$ by $\mathbf{K}$ to give $M$ theory on $\mathbf{J}_{\mathbf{K}}^{\mathbf{Z}_{\mathbf{n}}}$, a natural proposal is that this procedure commutes with the duality between $M$ theory on $\mathbf{C}^{\mathbf{2}} / \mathbf{Z}_{\mathbf{n}} \times \mathbf{T}^{\mathbf{3}}$ and the configuration of $n$ (wrapped) D6-branes in Type IIA theory. Hence, if this is the case, we expect a dual description 
of $M$ theory on $\mathbf{J}_{\mathbf{K}}^{\mathbf{Z}_{\mathbf{n}}}$ in terms of Type IIA theory on $\left(\mathbf{R}^{\mathbf{3}} \times \mathbf{T}^{\mathbf{3}}\right) / \mathbf{K}^{*}$ with $n$ D6-branes wrapped around the $\mathbf{T}^{\mathbf{3}}$ in the cover. Here $\mathbf{K}^{*}$ is the image of $\mathbf{K}$ under the map from $M$ theory to Type IIA theory. In particular, since $\mathbf{K}$ acts freely, the expectation that the Type IIA dual exists is fairly well justified [20].

Our next step will be to determine $\mathbf{K}^{*}$, and hence make a proposal for a Type IIA description of $M$ theory on $\mathbf{J}_{\mathbf{K}}^{\mathbf{Z}_{\mathbf{n}}}$.

The identification of how $\mathbf{K}^{*}$ acts on our wrapped D6-brane configuration in Type IIA theory on $\mathbf{R}^{\mathbf{3}} \times \mathbf{T}^{\mathbf{3}}$ follows from the simple observations: (i) the $S O(3)$ R-symmetry of the 7-dimensional super Yang-Mills theory acts via the 3 -dimensional representation on the coordinates of $\mathbf{R}^{\mathbf{3}}$; (ii) in $M$ theory on $\mathbf{J}_{\mathbf{1}}^{\mathbf{Z}_{\mathbf{n}}}$ it acts on the triplet of Kahler forms $\omega_{i}$ living on $\mathbf{C}^{\mathbf{2}} / \mathbf{Z}_{\mathbf{n}}$; (iii) in $M$ theory on $\mathbf{J}_{\mathbf{1}}^{\mathbf{Z}_{\mathbf{n}}} \mathbf{K}$ also acts (as a subgroup of $S O(3)$ ) on the triplet of 1-forms $d x_{i}$ in the same way as it does on the Kahler forms; (iv) since the $\mathbf{T}^{\mathbf{3}}$ is 'common' to both the Type IIA and $M$ theory backgrounds, this shows that $\mathbf{K}^{*}$ acts on the the coordinates of $\mathbf{T}^{\mathbf{3}}$ in the same way as $\mathbf{K}$. Combining these facts we learn that if the $\mathbf{R}^{\mathbf{3}}$ and $\mathbf{T}^{\mathbf{3}}$ have coordinates $v_{i}$ and $w_{i} \cong$ $w_{i}+1$ respectively, then the 1 -forms $d v_{i}$ and $d w_{i}$ transform under $\mathbf{K}^{*}$ in the 3 -dimensional representation. Moreover the coordinates $w_{i}$ transform under $\mathbf{K}^{*}$ in the same way as under $\mathbf{K}$.

It will be useful to denote the complex coordinates $Y_{i}$ as

$$
Y_{j} \equiv v_{j}+i w_{j}
$$

We can define a Calabi-Yau structure on $\mathbf{R}^{\mathbf{3}} \times \mathbf{T}^{\mathbf{3}}$ by defining the Kahler 2-form and holomorphic 3-form

$$
\begin{aligned}
\omega & =\frac{1}{2} \delta^{i j} d Y_{i} \wedge d Y_{j}^{*} \\
\Omega & =\frac{1}{6} \epsilon^{i j k} d Y_{i} \wedge d Y_{j} \wedge d Y_{k}
\end{aligned}
$$

These forms are clearly preserved by the $\mathbf{K}^{*}$ action on $\mathbf{R}^{\mathbf{3}} \times \mathbf{T}^{\mathbf{3}}$. In fact, they are manifestly $S O(3)$ invariant. Hence, the quotient space $\mathbf{Y}_{\mathbf{K}}$ $\equiv\left(\mathbf{R}^{\mathbf{3}} \times \mathbf{T}^{\mathbf{3}}\right) / \mathbf{K}^{*}$ admits a Calabi-Yau structure. Furthermore, since $\mathbf{K}^{*}$ acts freely on the $\mathbf{T}^{\mathbf{3}}$ factor, $\mathbf{Y}_{\mathbf{K}}$ is a smooth complex threefold, which (although its holonomy is not precisely $S U(3)$ ) we can regard as a Calabi-Yau threefold.

We are thus considering Type IIA string theory on $\mathbf{Y} \times \mathbf{R}^{4}$. This spacetime background preserves eight supercharges. We would now like to reintroduce our $n$ parallel D6-branes which fill $\mathbf{R}^{4}$. Since the D6-branes worldvolume is 7-dimensional, it must be of the form $\mathbf{M} \times \mathbf{R}^{4}$, where $\mathbf{M}$ is a real 
3-dimensional submanifold of $\mathbf{Y}_{\mathbf{K}}$. Furthermore, these D6-branes should be invariant under half of the eight supercharges preserved by the background, since we are ultimately interested in a configuration with $\mathcal{N}=1$ supersymmetry. This will be the case if, and only if, $\mathbf{M}$ is a special Lagrangian submanifold of $\mathbf{Y}_{\mathbf{K}}$. In fact, as we have discussed above, the D6-branes are sitting at the origin in the $\mathbf{R}^{\mathbf{3}}$ and are wrapped around the $\{\mathbf{0}\} \times \mathbf{T}^{\mathbf{3}}$ in the $\mathbf{R}^{\mathbf{3}} \times \mathbf{T}^{\mathbf{3}}$ covering space of $\mathbf{Y}_{\mathbf{K}}$. The image of this particular $\mathbf{T}^{\mathbf{3}}$ submanifold in $\mathbf{Y}_{\mathbf{K}}$ is simply $\left(\{\mathbf{0}\} \times \mathbf{T}^{\mathbf{3}}\right) / \mathbf{K}^{*}$ which we thus take as $\mathbf{M}$. This real 3-manifold is indeed a special Lagrangian submanifold of $\mathbf{Y}_{\mathbf{K}}$. Thus, we propose that the Type IIA configuration which is dual to $M$ theory on $\mathbf{J}_{\mathbf{K}}^{\mathbf{Z}_{\mathbf{n}}}$ is given by Type IIA string theory on $\mathbf{Y}_{\mathbf{K}}$ with $n$ D6-branes wrapped around the submanifold $\mathbf{M}$.

Let us now consider the Yang-Mills theory which describes the low energy world-volume dynamics of the D6-branes. We are thus considering 7-dimensional $S U(n)$ super Yang-Mills theory on $\mathbf{M} \times \mathbf{R}^{\mathbf{4}}$. Ordinarily, one would conclude that such a theory is not supersymmetric since $\mathbf{M}$ does not admit parallel spinors. However, $\mathbf{M}$ is a special Lagrangian submanifold in the Calabi-Yau threefold Y. and this fact implies that the super Yang-Mills theory is 'partially twisted' and indeed supersymmetric [19]. This will be explicitly illustrated shortly. Ultimately we are interested in the massless fields which propagate on $\mathbf{R}^{\mathbf{4}}$ and so we will think of this 7-dimensional Yang-Mills theory in a Kaluza-Klein fashion as being compactified from seven to four dimensions on $\mathbf{M}$.

In flat $\mathbf{R}^{\mathbf{7}}$, the symmetries of the Yang-Mills theory include $G \equiv \operatorname{Spin}(3) \times \operatorname{Spin}(6,1)$. The $\operatorname{Spin}(3)$ is the R-symmetry group and the $\operatorname{Spin}(6,1)$ the double cover of the spacetime Lorentz symmetry. The fields are all massless and consist of three real scalars $\phi_{i}$ which transforming as $(\mathbf{3}, \mathbf{1})$ under $G$, the gauge fields $A_{\mu}$, which transform as $(\mathbf{1}, \mathbf{7})$ under $G$, and two spacetime fermions transforming as $(\mathbf{2}, \mathbf{8})$. All of these fields transform in the adjoint representation of the gauge group $S U(n)$. Compactification of this theory on $\mathbf{M}$ to $\mathbf{R}^{\mathbf{4}}$ breaks $G$ to $G^{\prime} \equiv \operatorname{Spin}(3) \times \operatorname{Spin}(3)^{\prime} \times S L(2, \mathbf{C})$, where $S O(3)^{\prime} \equiv \operatorname{Spin}(3) / \mathbf{Z}_{\mathbf{2}}$ acts on the tangent bundle of $\mathbf{M}$ and $S L(2, \mathbf{C})$ acts on $\mathbf{R}^{\mathbf{4}}$ as the double cover of the Lorentz group. Under $G^{\prime}$, the scalars transform as $(\mathbf{3}, \mathbf{1}, \mathbf{1})$, the gauge fields split into a gauge field on $\mathbf{R}^{\mathbf{4}}$ transforming as $(\mathbf{1}, \mathbf{1}, \mathbf{2} \otimes \overline{\mathbf{2}})$ and three 'new' scalars $\phi_{i}^{\prime}$ transforming as $(\mathbf{1}, \mathbf{3}, \mathbf{1})$. The fermions transform as $(\mathbf{2}, \mathbf{2}, \mathbf{2})+(\mathbf{2}, \mathbf{2}, \overline{\mathbf{2}})$. As we remarked above, the lack of parallel spinors on $\mathbf{M}$ usually implies that the theory is not supersymmetric.

Now we must 'remember' that we are really discussing D6-branes wrapped around a supersymmetric cycle in a Calabi-Yau threefold. From this point 
of view, the scalars $\phi_{i}$ are re-interpreted as sections of the normal bundle $N(\mathbf{M})$ to $\mathbf{M}$ inside $\mathbf{Y}_{\mathbf{K}}$ and the scalars $\phi_{i}^{\prime}$ as sections of the tangent bundle $T(\mathbf{M})$ to $\mathbf{M}$. The fermion fields are sections of the spin bundle of $\mathbf{M}$ with values in the spin bundle constructed from the normal bundle. Thus far, we have not utilised the Calabi-Yau structure of $\mathbf{Y}_{\mathbf{K}}$. A remarkable observation due to Mclean [21] asserts that $T(\mathbf{M}) \cong N(\mathbf{M})$. In other words, the Calabi-Yau structure of $\mathbf{Y}_{\mathbf{K}}$ allows one to identify the normal bundle with the tangent bundle.

For our gauge theory, this is the 'origin' of the twisting which implies the theory is supersymmetric. Since the tangent and normal bundles to $\mathbf{M}$ have been identified, both sets of scalars can be considered as sections of $T(\mathbf{M})$. On the other hand, if $S(\mathbf{M})$ denotes the spin bundle of $\mathbf{M}$, the fermions can now be regarded as sections of $S \otimes S \cong \Lambda^{0}(\mathbf{M}) \oplus \Lambda^{1}(\mathbf{M}) \cong \Lambda^{0}(\mathbf{M}) \oplus T(\mathbf{M})$ In effect, all fields now transform under a group $G^{\prime \prime} \equiv S O(3)^{\prime \prime} \times S L(2, \mathbf{C})$, where $S O(3)^{\prime \prime}$ is again the structure group acting on the tangent space to M. Under $G^{\prime \prime}$ both sets of scalars transform as $(\mathbf{3}, \mathbf{1})$, the gauge fields as $(\mathbf{1}, \mathbf{2} \otimes \overline{\mathbf{2}})$. The fermions now transform as $(\mathbf{1}, \mathbf{2})+(\mathbf{1}, \overline{\mathbf{2}})+(\mathbf{3}, \mathbf{2})+(\mathbf{3}, \overline{\mathbf{2}})$. These latter fields are thus two spacetime spinors which are also scalars on $\mathbf{M}$ and two spacetime spinors which are 1-forms on $\mathbf{M}$.

We can finally compute the massless field content of the gauge theory on $\mathbf{R}^{\mathbf{4}}$. Fields on $\mathbf{R}^{\mathbf{4}}$ will be massless if they are also zero modes of the derivative operator which acts on them. The two four dimensional scalars are 1-forms on $\mathbf{M}$, so they will give rise to massless fields if the (free part of the) first cohomology group of $\mathbf{M}$ is non-trivial. Since we have two such scalars they will contribute $2 b_{1}$ scalars to the four dimensional theory. The four dimensional gauge fields are zero-forms on $\mathbf{M}$ so, since $b_{o}$ is 1 , it gives rise to one massless four dimensional gauge field. The spinors split into zeroforms and 1-forms on $\mathbf{M}$, and they contribute $\left(1+b_{1}\right)$ massless fermions to the theory. Also, due to the fact that the compactification on $\mathbf{M}$ preserves the $S U(n)$ gauge symmetry, all these fields take values in the Lie algebra of $S U(n)$. The four dimensional massless field content therefore consists of one gauge field, $2 b_{1}$ scalars and $1+b_{1}$ fermions which all transform in the adjoint of the $S U(n)$ gauge group. How many supersymmetries exist in the four dimensional theory ? The supersymmetries of the theory are in one to one correspondence with the fermion zero modes. These number $1+b_{1}$.

Since the first Betti number of $\mathbf{M}$ is zero, we find that the massless field content is precisely that of $\mathcal{N}=1$ super Yang-Mills theory with gauge group $S U(n)$.

Note that classically $\mathcal{N}=1$ super Yang-Mills theory has a $U(1) \mathrm{R}$ - 
symmetry group. $\operatorname{Spin}(3)$ symmetries are nowhere to be seen in that theory. How is this fact reconciled with the statement of the previous paragraph? Firstly, the supersymmetries and massless fields are all singlets under the Spin(3) action, so in the theory of interest, this group acts trivially. Secondly, the $U(1)$ R-symmetry of the theory is also present - it is simply not obvious in the way we have described the theory. This can easily been seen by noting that an R-symmetry acts on the superspace coordinates. In fact the superspace measure $d^{2} \theta$ transforms under $U(1)$ R-symmetries with a charge minus that of the holomorphic 3 -form $\Omega$. In fact, if we canonically normalise so that under a $U(1)$ rotation $d^{2} \theta$ transforms with charge -2 , then the $U(1)$ R-symmetry of the model acts on the coordinates of $\mathbf{Y}$ as follows:

$$
U(1): Y_{j} \rightarrow e^{\frac{2}{3} i \alpha} Y_{j}
$$

This is clearly not a manifest symmetry of the wrapped D6-brane configuration. Therefore, the symmetries of the Yang-Mills theory are not obviously in contradication to those of the D6-branes. wrapped on M. Suppose, for arguments sake, that the D6-branes were wrapped around a special Lagrangian submanifold $\mathbf{M}^{\prime}$ in some other Calabi-Yau threefold $\mathbf{Y}^{\prime}$. Further suppose that $b_{1}\left(\mathbf{M}^{\prime}\right)=1$. Then, according to the above analysis, the effective four dimensional theory has $\mathcal{N}=2$ supersymmetry. Classically, a field theory with these supersymmetries has an $\operatorname{Spin}(3) \times U(1)$ R-symmetry group. However, in the D6-brane model, only the $\operatorname{Spin}(3)$ symmetry is obvious.

\section{$4 \quad \mathrm{~N}=1$ Super Yang-Mills}

For completeness and in order to compare easily with $M$ theory results obtained later we briefly give a review of $\mathcal{N}=1$ pure super Yang-Mills theory. More detailed reviews can be found in [15]. We begin with gauge group $S U(n) . \mathcal{N}=1 S U(n)$ super Yang-Mills theory in four dimensions is an extensively studied quantum field theory. The classical Lagrangian for the theory is

$$
\mathcal{L}=-\frac{1}{4 g^{2}}\left(F_{\mu \nu}^{a}\right)^{2}+\frac{1}{g^{2}} \bar{\lambda}^{a} i \not \mathcal{D} \lambda^{a}+i \frac{\theta}{32 \pi^{2}} F_{\mu \nu}^{a} \tilde{F}^{a \mu \nu}
$$

$F$ is the gauge field strength and $\lambda$ is the gaugino field.

It is widely believed that this theory exhibits dynamics very similar to those of ordinary QCD: confinement, chiral symmetry breaking, a mass gap. Supersymmetry constrains the dynamics of the theory so strongly, that the low energy effective superpotential for the model is known. It is given by

$$
W_{e f f}=c \mu^{3} e^{2 \pi i \tau / n}
$$


here $\tau$ is the complex coupling constant,

$$
\tau=\frac{\theta}{2 \pi}+i \frac{4 \pi}{g^{2}}
$$

and $\mu$ the mass scale.

In particular, the form of this potential suggests that it is generated by dynamics associated with "fractional instantons", ie states in the theory whose quantum numbers are formally of instanton number $\frac{1}{n}$. Such states are also closely related to the spontaneously broken chiral symmetry of the theory. Let us briefly also review some of these issues here.

Under the $U(1)$ R-symmetry of the theory, the gauginos transform as

$$
\lambda \rightarrow e^{i \alpha} \lambda
$$

This is a symmetry of the classical action but not of the quantum theory (as can easily be seen by considering the transformation of the fermion determinant in the path integral). However, if the above transformation is combined with a shift in the theta angle of the form

$$
\tau \rightarrow \tau+\frac{2 n}{2 \pi} \alpha
$$

then this cancels the change in the path integral measure. This shift symmetry is a bona fide symmetry of the physics if $\alpha=\frac{2 \pi}{2 n}$, so that even in the quantum theory a $\mathbf{Z}_{\mathbf{2 n}}$ symmetry remains. Associated with this symmetry is the presence of a non-zero value for the following correlation function,

$$
\left\langle\lambda \lambda\left(x_{1}\right) \lambda \lambda\left(x_{2}\right) \ldots \lambda \lambda\left(x_{n}\right)\right\rangle
$$

which is clearly invariant under the $\mathbf{Z}_{\mathbf{2 n}}$ symmetry. This correlation function is generated in the 1-instanton sector and the fact that $2 n$ gauginos enter is due to the fact that an instanton of charge 1 generates $2 n$ chiral fermion zero modes.

Cluster decomposition implies that the above correlation function decomposes into ' $n$ constituents' and therefore there exists a non-zero value for the gaugino condensate:

$$
\langle\lambda \lambda\rangle \neq 0
$$

Such a non-zero expectation value is only invariant under a $\mathbf{Z}_{\mathbf{2}}$ subgroup of $\mathbf{Z}_{2 \mathbf{n}}$ implying that the discrete chiral symmetry has been spontaneously broken. Consequently this implies the existence of $n$ vacua in the theory. 
In fact, it can be shown that

$$
\langle\lambda \lambda\rangle=16 \pi i \frac{\partial}{\partial \tau} W_{e f f}=-\frac{32 \pi^{2}}{n} c \mu^{3} e^{2 \pi i \tau / n}
$$

In view of the above facts it is certainly tempting to propose that 'fractional instantons' generate the non-zero gaugino condensate (18) directly. But this is difficult to see directly in SQCD. We will see that this is precisely what the dynamics of $M$ theory on $\mathbf{J}_{\mathbf{K}}^{\mathbf{z}_{\mathbf{n}}}$ predicts.

More generally, if we replace the $S U(n)$ gauge group by some other gauge group $H$, then the above statements are also correct but with $n$ replaced everywhere with $c_{2}(H)$ the dual Coxeter number of $H$. For A-D-E gauge groups $c_{2}(H)=\Sigma_{i=1}^{r+1} a_{i}$, where $r$ is the rank of the gauge group and the $a_{i}$ are the Dynkin indices of the affine Dynkin diagram associated to $H$. For $A_{n}$, all the $a_{i}=1$; for $D_{n}$ groups the four 'outer' nodes have index 1 whilst the rest have $a_{i}=2$. $E_{6}$ has indices $(1,1,1,2,2,2,3), E_{7}$ has $(1,1,2,2,2,3,3,4)$ whilst $E_{8}$ has indices $(1,2,2,3,3,4,4,5,6)$.

\section{Theta angle and Coupling Constant in $M$ theory}

The physics of $M$ theory supported near the singularities of $\mathbf{C}^{\mathbf{2}} / \mathbf{G} \times \mathbf{R}^{\mathbf{7}}$ is described by super Yang-Mills theory on $\mathbf{R}^{\mathbf{7}}$. The gauge coupling constant of the theory is given by

$$
\frac{1}{g_{7 d}^{2}} \sim \frac{1}{l_{p}^{3}}
$$

where $l_{p}$ is the eleven dimensional Planck length. In seven dimensions, the analog of the theta angle in four dimensions is actually a three-form $\Theta$. The reason for this is the seven dimensional interaction

$$
L_{I} \sim \Theta \wedge F \wedge F
$$

(with $F$ the Yang-Mills field strength). In $M$ theory $\Theta$ is given by $A$, the three-form potential for the theory.

If we now take $M$ theory on $\mathbf{J}_{\mathbf{K}}^{\mathbf{G}}$ we have essentially 'compactified the seven dimensional theory with a twist' and the four dimensional gauge coupling constant is roughly given by

$$
\frac{1}{g_{4 d}^{2}} \sim \frac{V_{M}}{l_{p}^{3}}
$$


where $V_{M}$ is the volume of $\mathbf{M}$. The four dimensional theta angle can be identified as

$$
\theta=\int_{\mathbf{M}} A
$$

The above equation is correct because under a global gauge transformation of $A$ which shifts the above period by $2 \pi$ times an integer - a transformation which is a symmetry of $M$ theory - $\theta$ changes by $2 \pi$ times an integer. Such shifts in the theta angle are also global symmetries of the field theory.

Thus the complex gauge coupling constant of the effective four dimensional theory may be identified as

$$
\tau=\int_{\mathbf{M}}\left(\frac{A}{2 \pi}+i c \frac{\varphi}{l_{p}^{3}}\right)
$$

( $c$ is a normalisation constant) The quantities on the right hand side in the above expression are the real and imaginary parts of a complex scalar field in the theory which is the bosonic part of the single massless chiral superfield in the background $\mathbf{J}_{\mathbf{K}}^{\mathbf{G}}$. (The reason that there is only one massless chiral superfield stems from the fact that $b_{3}^{s t r}$, the third string theoretic Betti number of $\mathbf{J}_{\mathbf{K}}^{\mathbf{G}}$ is equal to one.) In view of this we should really view $\tau$ as a background superfield and regard the above equation as an expression for its vacuum expectation value.

\section{BPS States}

In any $\mathcal{N}=1$ supersymmetric theory, a natural class of states to discuss are the BPS saturated states. These are states which are invariant under two of the four supercharges. In general, such a theory can contain BPS instantons, strings and domain walls. However, in the pure $\mathcal{N}=1$ super Yang-Mills theory which interests us, only BPS instantons and domain walls can appear. A simple and natural question to pose is where are these states in the $M$ theory and Type IIA backgrounds we have been discussing?

The instantons will be of primary interest to us in this paper.

\subsection{M2-brane Instantons}

In $M$ theory on $\mathbf{C}^{\mathbf{2}} / \mathbf{G} \times \mathbf{R}^{\mathbf{7}}$, the $M$ theory state which corresponds to an instanton of charge one in the super Yang-Mills theory is one M2-brane whose world-volume is of the form $\mathbf{R}^{\mathbf{3}} \subset \mathbf{R}^{\mathbf{7}}$. We can think of this charge one 
$M 2$-brane as $|\mathbf{G}| \mathbf{G}$-equivariant $M 2$-branes on $\mathbf{C}^{\mathbf{2}} \times \mathbf{R}^{\mathbf{7}}$. However, because $\mathbf{G}$ does not act on the $\mathbf{R}^{4}$ which is transverse to the world-volume of the $M 2$-branes in spacetime, these $|\mathbf{G}| M 2$-branes can be at different positions in spacetime. From the point of view of a low energy field theory observer in $\mathbf{R}^{\mathbf{7}}$, each of these branes carry fractional instanton number. These are just the fractional branes described in [12].

The precise amount of charge carried by these branes is naturally encoded in the extended Dynkin diagram associated with the A-D-E singularity. As explained in [22], the dynamics of a charge one D-brane moving on $\mathbf{C}^{2} / \mathbf{G}$ is described by a quiver gauge theory on the world-volume whose quiver diagram is related to the affine Dynkin diagram of the A-D-E group. Let $a_{i}$ denote the Dynkin index for the $i$ 'th node of such a diagram; then the gauge group of the theory is $\Pi_{i} U\left(a_{i}\right)$ which means it is natural to associate $a_{i}$ D-branes to the $i$ 'th node. Since

$$
\Sigma_{i=1}^{r+1} a_{i}=c_{2}(H)
$$

where $r$ is the rank of the group $H$, the single D-brane 'fractionates' into $r+1$ fractional D-branes the $i$ 'th of which carries charge $\frac{a_{i}}{c_{2}(H)}$. In the $M$ theory lift of this structure that we wish to consider here, with the D-brane replaced by the $M 2$-brane, it is natural to assume that the same fractionation of charge takes place.

For example, in the case when $\mathbf{G}$ is $\mathbf{Z}_{\mathbf{n}}$ so that the gauge group is $S U(n)$, all the $n$ nodes have index 1 and the single $M 2$-brane instanton fractionates into $n$ identical fractional instantons each of charge $\frac{1}{n}$.

Now consider the case when we geometrically engineer using $\mathbf{J}_{\mathbf{K}}^{\mathbf{G}}$. The $M$ theory states which correspond to field theory instantons now correspond to $M 2$-branes wrapped around the supersymmetric 3-cycle $\mathbf{M}_{\mathbf{K}}$. However, the group $\mathbf{G}$ is still acting transverse to these $M 2$-branes and hence one can see that the BPS spectrum of $M$ theory on $\mathbf{J}_{\mathbf{K}}^{\mathbf{G}}$ contains fractional $M 2$-brane instantons.

We have argued that $M$ theory on $\mathbf{J}_{\mathbf{K}}^{\mathbf{G}} \times \mathbf{R}^{4}$ gives rise to a geometrically engineered version of super Yang-Mills theory with four supercharges and A-D-E gauge group. Furthermore, we have identified instantons of the field theory as $M 2$-branes in $M$ theory. Since in the super Yang-Mills theory in question, a charge one instanton generates $2 c_{2}(H)$ chiral fermionic zero modes, we can fairly safely assume that the corresponding charge one wrapped $M 2$-brane also generates precisely $2 c_{2}(H)$ such zero modes. This has the following simple consequence: a fractional $M 2$-brane instanton of charge $\frac{1}{c_{2}(H)}$ generates two fermionic zero modes. This implies that they can 
in principle generate a superpotential in the four dimensional theory. If such a superpotential is generated, it will be of the form,

$$
W=C \cdot e^{-S_{I}}
$$

where $S_{I}$ is the supersymmetric action for the $M 2$-brane instanton (and $C$ is a dimensionful normalisation constant). $S_{I}$ will contain a contribution proportional to the volume of the $M 2$-brane world-volume (ie $\mathbf{M}$ ), with a constant of proportionality determined by the topological charge.

In fact

$$
W=C \cdot e^{2 \pi i \tau / c_{2}(H)}
$$

The reason that the exponent comes precisely with the above factor is that $\exp \left(-S_{I}\right)$ for an instanton of charge one must be given by

$$
e^{2 \pi i \tau}
$$

This is because the real part of the exponent is minus the action for one instanton and the superpotential must be holomorphic. A convenient way of writing (27) is

$$
C^{\prime} \cdot e^{2 \pi i \tau}=\Pi_{i=1}^{r+1} W^{a_{i}}
$$

Hence, we can interpret $W^{a_{i}}$ as $\exp \left(-S_{I}^{a_{i}}\right)$, where $S_{I}^{a_{i}}$ is the action for a fractional instanton of charge $\frac{a_{i}}{c_{2}(H)}$. Thus we see that equation (26) is just the action for those fractional instantons associated with nodes of index 1 (ie with topological charge $\left.\frac{1}{c_{2}(H)}\right)$.

Clearly the result (26) agrees with the field theory expectation (12). Moreover the fact that the superpotential is dynamically generated by an object carrying a fractional instanton number also agrees with the results of $[16,17,13,18]$.

\subsection{Relation to Membrane Anomalies}

In field theory instantons are usually associated with anomalies. Whether or not the $M 5$-brane instantons considered in [23] generated a superpotential was shown to be related to a certain anomaly in rotations of the normal bundle to the $M 5$-brane. On the other hand, $M 2$-branes have odd dimensional world-volumes and the only known anomaly associated with them is the so-called parity anomaly on the world-volume [24]. This can occur if the world-volume of the $M 2$-brane is transported around a transverse circle. 
For a charge one $M 2$-brane with world-volume $\mathbf{M}$, the path integral for $M$ theory includes the factor

$$
e^{i \int_{\mathbf{M}} A}
$$

The change in this factor as the world-volume is transported around a transverse circle $\mathbf{S}^{\mathbf{1}}$ is

$$
e^{i \int_{\mathbf{M} \times \mathbf{S}^{1}}(\lambda \pi+G)}
$$

where $\lambda$ is a certain characteristic class [24] and $G$ is the field strength of $A$. Since $2 \pi R e \tau=\int_{\mathbf{M}} A$, from the point of view of our geometrically engineered theory, the above change can be interpreted as a shift in the theta angle

$$
\tau \rightarrow \tau+\frac{\int_{\mathbf{M} \times \mathbf{S}^{\mathbf{1}}}(\lambda \pi+G)}{2 \pi}
$$

If we compare to field theory, then (at least for certain choices of circles around which the membrane is transported) it is natural to try and identify this rotation as a consequence of an R-symmetry transformation. If we let

$$
e^{i \int_{\mathbf{M} \times \mathbf{S}^{1}}(\lambda \pi+G)}=e^{i k \alpha}
$$

we wish to determine the 'charge' $k$. We can determine $k$ as follows. In field theory, if we normalise the R-symmetry generator as in (14) so that the gauginos transform with charge one, then the superpotential transforms with charge two. Equation (28) then implies that

$$
k=2 \Sigma_{i} a_{i}=2 c_{2}(H)
$$

With the charge determined we see that equation (31) becomes

$$
\tau \rightarrow \tau+\frac{2 c_{2}(H)}{2 \pi} \alpha
$$

This agrees precisely with the field theory result (15). Hence if our assumptions about the R-symmetry are correct $M$ theory "knows" that the chiral symmetry is $\mathbf{Z}_{\mathbf{2} \mathbf{c}_{\mathbf{2}}(\mathbf{H})}$ since the cancellation of the membrane anomalies implies that (34) is a symmetry. The generation of the superpotential (26) then implies that this chiral symmetry is spontaneously broken to $\mathbf{Z}_{\mathbf{2}}$ by fractional $M 2$-brane instantons. This is a rather satisfying result. 


\section{Acknowledgements.}

The author would like to thank Steve Thomas for comments, E. Witten for discussions in 1996 concerning membrane instanton generated superpotentials and G. Moore for informing us about [25] prior to publication. We would also like to acknowledge discussions with D. Morrison and R. Plesser during which we realised the existence of non-trivial monodromies in certain examples. The author is supported by a PPARC Postdoctoral Fellowship.

\section{References}

[1] A. Hanany and E. Witten, Type IIB Superstrings, BPS Monopoloes and Three Dimensional Gauge Dynamics, Nucl. Phys. B492 (1997) 152-190; hep-th/9611230.

[2] A. Giveon and D. Kutasov, Brane Dynamics and Gauge Theory, hepth/9802067.

[3] S. Katz, A. Klemm and C. Vafa, Geometric Engineering of Quantum Field Theories, Nucl. Phys. B497 (1997) 173; hepth/9609239

[4] P. Aspinwall, K3 Surfaces and String Duality, hep-th/9611137.

M. Bershadsky, K. Intrilligator, S. Kachru, D. Morrison, V. Sadov and C. Vafa, Geometric Singularities and Enhanced Gauge Symmetries, Nucl. Phys. B481 (1996) 215; hep-th/9605200.

[5] G. Papadopoulos and P. Townsend, Compactification of $d=11$ Supergravity on Manifolds of Exceptional Holonomy, Phys. Lett. B357 (1995) 300 .

[6] S. Katz and C. Vafa, Geometric Engineering of $\mathrm{N}=1$ Quantum Field Theories, Nucl. Phys.B497 (1997) 196; hep-th/9611090

M. Bershadsky, A. Johansen, T. Pantev, V. Sadov and C. Vafa, F-theory, Geometric Engineering and N=1 Dualities, Nucl. Phys. B505 (1997) 153; hep-th/9612052

C. Vafa and B. Zweibach, N=1 Dualities of SO and USp Gauge Theories and T-duality of String Theory, Nucl. Phys. B506 (1997) 143; hepth/971015.

H. Ooguri and C. Vafa, Geometry of $\mathrm{N}=1$ Dualities in Four Dimensions, Nucl. Phys. B500 (1997) 62; hep-th/9702180.

C. Vafa, On $\mathrm{N}=1$ Yang-Mills in Four Dimensions, ATMP. 2 (1998) 497;hep-th/9801139 
[7] D. Joyce, Compact Riemannian 7-manifolds With Holonomy $G_{2}$. II J. Diff. Geom. 43, 2 (1996) 329.

[8] J. Harvey, D. Lowe and A. Strominger, N=1 String Duality, Phys. Lett. B362 (1995) 65; hep-th $/ 9507168$

B.S. Acharya, $\mathrm{N}=1$ Heterotic/M theory Duality and Joyce Manifolds, Nucl. Phys. B475 (1996) 579; hep-th/9603033

[9] Chien-Hao Liu, On the Global Structure of Some Natural Fibrations of Joyce Manifolds, hep-th/9809007.

[10] S. Katz, D. Morrison and M. Plesser Enhanced Gauge Symmetrx in Type II String Theory, Nucl. Phys. B477 (1996) 105; hep-th/9601108.

[11] P. Aspinwall, Enhanced Gauge Symmetries and Calabi-Yau Threefolds, Phys. Lett. B371 (1997) 231; hep-th/9511171

[12] D. Diaconescu, M. Douglas and J. Gomis, Fractional Branes and Wrapped Branes, JHEP O2 (1998) 13; hep-th9712230.

[13] J. Brodie, Fractional Branes, Confinement and Dynamically Generated Superpotentials Nucl. Phys. B532 (1998) 137; hep-th/9803140.

[14] K. Hori, H. Ooguri and Y. Oz, Strong Coupling Dynamics of Four Dimensional $\mathrm{N}=1$ Gauge Theory fro $\mathrm{M}$ theory Fivebrane, ATMP 1 (1998) 1; hep-th/9706082.

E. Witten, Branes and The Dynamics of QCD, Nucl. Phys. B507 (1997) 658; hep-th/9706109.

[15] D. Amati et. al, Non-Perturbative Aspects of Superymmetric Gauge Theories, Phys. Rep. B162 (1988) 169.

M. Shifman, Non-Perturbative Dynamics in Supersymmetric Gauge Theories hep-th/9704114

K. Intrilligator and N. Seiberg, Lectures on Supersymmetric Gauge Theories and Electric-Magnetic Duality, Nucl. Phys. Proc. Suppl. 45BC (1996) 1; hep-th/9506084.

M. Peskin, Duality in Supersymmetric Yang-Mills Theory hep-th/9702094.

[16] E. Cohen and C. Gomez, Chiral Symmetry Breaking in Supersymmetric Yang-Mills, Phys. Rev. Lett. 52 (1984) 237.

[17] C. Gomez and R. Hernandez, M and F-theory Instantons, N=1 Supersymmetry and Fractional Topological Charges, Int. J. Mod. Phys. A12 (1997) 5141; hep-th 9701150.

[18] M. Zabzine, Fractional Instantons in Supersymmetric Gauge Theories, hepth/9807120. 
[19] M. Bershadsky, V. Sadov and C. Vafa, D-branes and Topological Field Theories Nucl. Phys. B463 (1996) 420; hep-th/9511222.

[20] A. Sen, Duality and Orbifolds, Nucl. Phys. B474 (1996) 361; hep-th 9604070 .

[21] R. Mclean, Deformations of Calibrated Submanifolds, Ph.D. Thesis (1996)

[22] M. Douglas and G. Moore, D-branes, Quivers and ALE Instantons, hepth/9603167.

C. Johnson and R. Myers, Aspects of Type IIB Theory on ALE Spaces, Phys. Rev. D55 (1997) 6382; hep-th/9610140.

[23] E. Witten, Non-Perturbative Superpotentials in String Theory, Nucl. Phys. B474 (1996) 343; hep-th/9604030.

[24] E. Witten, Flux Quantisation in $\mathrm{M}$ theory and The Effective Action, $J$. Geom. Phys. 22 (1997) 1; hep-th/9609122.

[25] J. Harvey and G. Moore, In Preparation. 